Gosselin S, Morris M, Miller-Nesbitt A, Hoffman RS, Hayes BD, Turgeon AF, Gilfix BM, Grunbaum AM, Bania TC, Thomas SH, Morais JA, Graudins A, Bailey B, Megarbane B, Calello DP, Levine M, Stellpflug SJ, Hoegberg LC, Chuang R, Stork C, Bhalla A, Rollins CJ, Lavergne V. Methodology for AACT evidence-based recommendations on the use of intravenous lipid emulsion therapy in poisoning. Clinical Toxicology 2015, 53(6), 557-564

\section{Copyright:}

This is an Accepted Manuscript of an article published by Taylor \& Francis in Clinical Toxicology on 10/6/2015, available online: http://www.tandfonline.com/10.3109/15563650.2015.1052498.

DOI link to article:

http://dx.doi.org/10.3109/15563650.2015.1052498

Date deposited:

$08 / 06 / 2016$

Embargo release date:

10 June 2016

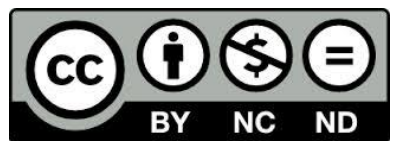

This work is licensed under a

Creative Commons Attribution-NonCommercial-NoDerivatives 4.0 International licence 


\section{AACT Evidence-based recommendations on the use of}

\section{Lipid Emulsion Therapy in poisoning methodology}

\section{Authors}

1. Sophie Gosselin, MD. Department Of Emergency Medicine, Medical Toxicology Consultation Service, McGill University Health Centre, and Centre Antipoison du Québec, Québec, Québec, Canada.

2. Martin Morris, MSc. Liaison Librarian, Life Sciences Library, McGill University, Montréal, Québec, Canada.

3. Andrea Nesbitt-Miller, MLIS. Liaison Librarian, Life Sciences Library, McGill University, Montréal, Québec, Canada.

4. Robert S. Hoffman, MD. Division of Medical Toxicology, Ronald O. Perelman Department of Emergency Medicine, New York University School of Medicine, New York, New York, USA.

5. Bryan D. Hayes, PharmD. Department of Pharmacy, University of Maryland Medical Center and Department of Emergency Medicine, University School of Medicine, Baltimore, Maryland, USA.

6. Alexis F. Turgeon, MD MSc. Division of Critical Care Medicine, Department of Anesthesiology and Critical Care Medicine, and CHU de Québec Research Center, Population Health and Optimal Health Practices Unit, Université Laval, Québec City, Québec, Canada.

7. Brian M. Gilfix, MD PhD. Division of Medical Biochemistry, Department of Medicine, McGill University Health Centre, Montreal, Québec, Canada.

8. Ami M. Grunbaum, MD. Division of Medical Biochemistry, Department of Medicine. McGill University Health Centre, Montréal, Québec, Canada.

9. Theodore C. Bania, MD. Mt Sinai Roosevelt, Mt Sinai St. Luke's, Department of Emergency Medicine, Icahn School of Medicine at Mt Sinai, New York, New York, USA.

10. Simon H.L. Thomas, MD. National Poisons Information Service (Newcastle) and Medical Toxicology Centre, Institute of Cellular Medicine, Newcastle University, Newcastle, UK.

11. José A. Morais, MD. Department of Medicine, Crabtree Nutrition Laboratories, McGill University Health Centre, McGill University, Montreal, Québec, Canada.

12. Andis Graudins, MB BS PhD. Monash Emergency Medicine and Clinical Toxicology, Monash Health and Southern Clinical School, Faculty of Medicine, Nursing and Health Sciences, Monash University, Clayton, Victoria, Australia.

13. Benoit Bailey, MD MSc, Division of Emergency Medicine Department of Pediatrics, CHU SainteJustine, Montréal, Québec Canada, Centre Antipoison du Québec, Québec, Canada.

14. Bruno Mégarbane, MD. Department of medical and toxicological intensive care, Lariboisière Hospital, Paris-Diderot University, Paris, France. 
15. Diane P. Calello, MD. Medical Toxicology, Department of Emergency Medicine, Morristown Medical Center, Emergency Medical Associates, Morristown, New Jersey, USA.

16. Michael Levine, MD. Department of Emergency Medicine, Section of Medical Toxicology. University of Southern California. Los Angeles, California, USA.

17. Samuel J. Stellpflug, MD. Department of Emergency Medicine, Regions Hospital, Saint Paul, Minnesota, USA.

18. Lotte C.G. Hoegberg, PhD. Danish Poisons Information Centre, Anesthesiology, Bispebjerg University Hospital, Copenhagen, Denmark.

19. Ryan Chuang, MD. University of Calgary, Department of Emergency Medicine, Division of Clinical Pharmacology and Toxicology, Poison and Drug Information Service, Calgary, Alberta, Canada.

20. Christine Stork, PharmD. Upstate NY Poison Center and Department of Emergency Medicine, Upstate Medical University. Syracuse, New York, USA.

21. Ashish Bhalla, MD. Department of internal medicine, Post Graduate Institute of medical Education and Research, Sector 12, Chandigarh, India.

22. Carol J. Rollins, MS PharmD BCNSP. University of Arizona Medical Center, University of Arizona College of Pharmacy, Tucson, Arizona, USA.

23. Valéry Lavergne, MD MSc, Department Of Medical Biology, Sacré-Coeur Hospital, University of Montréal, Montréal, Québec, Canada.

\section{On behalf of the AACT Lipid Emulsion Therapy workgroup*}

*The AACT Lipid Emulsion Therapy workgroup also consists of Marjorie BonHomme, MD and Sheldon Magder, MD.

\section{Keywords}

Intravenous lipid emulsion, fat emulsion, lipid resuscitation, recommendation, efficacy, adverse effect, lipid interference

Words 3603

Tables 3, Figure 1, Appendices 5

\section{Corresponding author}

Dr Valéry Lavergne 
5400 Boulevard Gouin Ouest,

Montréal, Québec, Canada

H4J 1C5

Tel : 514-338-2222

Fax : 514-338-3307 


\title{
Methodology for AACT Evidence-based Recommendations on the use of Intravenous Lipid Emulsion Therapy in poisoning
}

\begin{abstract}
Intravenous lipid emulsion (ILE) therapy is a novel treatment that was discovered in the last decade. Despite unclear understanding of its mechanisms of action, numerous and diverse publications attested to its clinical use. However, current evidence supporting its use is unclear and recommendations are inconsistent. To assist clinicians in decisionmaking, the American Academy of Clinical Toxicology created a workgroup composed of international experts from various clinical specialties, which includes representatives of major clinical toxicology associations. Rigorous methodology using the AGREE II instrument was developed to provide a framework for the systematic reviews for this project and to formulate evidence-based recommendations on the use of ILE in poisoning. Systematic reviews on the efficacy of ILE in local anesthetic toxicity and nonlocal anesthetic poisonings as well as adverse effects of ILE are planned. A comprehensive review of lipid analytical interferences and a survey of ILE costs will be developed. The evidence will be appraised using the GRADE system. A thorough and transparent process for consensus statements will be performed to provide recommendations, using a modified Delphi method with two rounds of voting. This process will allow for the production of useful practice recommendations for this therapy.
\end{abstract}




\section{Introduction}

Local anesthetic systemic toxicity (LAST) is a potentially severe iatrogenic occurrence with an estimated incidence of approximately 1 per 1000 procedures. The clinical presentation ranges from seizures and dysrhythmias to cardiac arrest. Treatment consisting of oxygenation, ventilation, atropine and standard cardiac resuscitation, is often ineffective (1). Overdoses with lipid-soluble substances, such as antidepressants and cardiac medications, rank $5^{\text {th }}$ and $6^{\text {th }}$ in the list of most common substance categories reported to the US National Poison Data System (2). Although the fatality rate from the data available remains imprecise, neurotoxicity and cardiovascular toxicity associated with lipid soluble substances may be severe and significant morbidity is common.

A brief historical review of the use of intravenous lipid emulsion (ILE) in medical toxicology starts in 1997, when an in-vivo animal model of bupivacaine toxicity showed that in the presence of ILE the amount of bupivacaine required to induce LAST was significantly increased (3). It is almost a decade later, in 2006, that the first human case of LAST successfully treated with ILE therapy was reported (4). In 2008, with the hypothesis that other lipid soluble drugs might also be influenced by this treatment, ILE was used in a patient with oral bupropion and lamotrigine toxicity after standard toxicology resuscitation was failing, with subsequent survival (5). Since the publication of this case report, ILE has been increasingly employed in anesthesia and medical toxicology $(4,5)$. The American College of Medical Toxicology published an interim guidance statement regarding its use in 2011 (6). Despite many reviews published in 
recent years, the literature is dominated by animal models with unclear generalizability to humans, numerous opinion articles, and anecdotal case reports of variable quality and highly prone to publication bias (7-9).

Previously, ILE was primarily used for parenteral nutrition, drug transport or chemoembolization. Currently, they have become part of the treatment options available to anesthesiologists, intensivists, emergency physicians, and medical toxicologists in the treatment of poisoning. Although the mechanisms of actions are still not entirely clear, three main hypotheses have been proposed: sequestration of the offending xenobiotic in an expanded plasma lipid phase (the "lipid sink" theory), alteration in ionic channel permeability (activation of voltage-gated calcium or sodium channels) and modification of fatty acid utilization by the poisoned myocardium (the "bioenergetics" theory)(10).

The present workgroup was established by the American Academy of Clinical Toxicology to produce evidence-based recommendations on the use of ILE in poisoning to aid clinicians in their decision-making in urgent situations. Recommendations are intended to rely on the best available data, although few controlled studies have been conducted on the use of ILE in humans. The non-standardized protocols used for ILE result in significant variability in management, both in amount of ILE given as well as in duration of treatment. The rapid clinical improvements reported in some published case reports have therefore fostered an enthusiasm for this new therapy, especially for patients who fail to respond to other treatments. Frequently, the dose and duration of ILE for treatment of lipophilic drug toxicity exceed the maximum accepted dosage used in the 
parenteral nutrition literature. Potential adverse effects and costs have neither been well studied nor reported.

There are numerous factors that create variability, and therefore pose difficulties in comparing studies. These factors include the large number of xenobiotics for which this therapy has been tried, inconsistencies in the amount, duration and constitution of ILE used, and the differences between oral poisoning and LAST, which is more often parenteral. Nevertheless, this workgroup has elected to proceed with the systematic identification, review and critique of all available evidence using the Grading of Recommendations Assessment, Development and Evaluation (GRADE) approach(11). Because human case reports may reveal important information about toxicokinetics, and because there appears to be a temporal relationship between ILE administration and clinical improvement, this workgroup has opted to include case reports.

With the anticipated low quality evidence, the term "guidelines" might not be appropriate for this endeavor. Nevertheless, because of the use of the Appraisal of Guidelines for Research and Evaluation (AGREE) II instrument (Appendix 1 available on-line at: insert URL here), the results are based on a rigorous process of synthesis and methodology, which under the circumstances adds value to the current debate (12). The workgroup has therefore chosen the wording "Recommendations", which will reflect the best available evidence at the time of publication. 


\section{Objectives of the workgroup}

Our objectives are to summarize the available evidence on: 1) the clinical efficacy of ILE with non-local anesthetic poisoning; 2) the clinical efficacy of ILE with local anesthetic poisoning; and 3) the adverse effects of ILE divided into clinical effects and analytical interference. To fulfill these objectives, three systematic reviews and a comprehensive review on analytical interferences from ILE will be performed. Based on the evidence collected and the results of these systematic and comprehensive reviews, recommendations on the use of ILE in the context of poisoning will be developed. The clinical efficacy of ILE will be measured against current standards of care and alternative treatments, and balanced with its potential complications and costs.

\section{Methodology}

\section{Group selection}

At least two representatives from national and international medical toxicology associations have been delegated by their respective boards to serve on this workgroup. The number of representatives was selected according to the size of the associations' membership. In order to ensure that the workgroup was fully representative, and included different specialty stakeholders with the expertise needed to assess every aspect of this therapy, additional experts were recruited based on recommendations from various societies or on their particular clinical expertise. One participant was chosen because of expertise in epidemiology and guideline methodology, but will not participate in the voting of the recommendations. Two medical librarians were included in the workgroup to design and conduct systematic searches of the literature and to 
assist with article retrieval but were not voting members. Medical biochemists and nonclinician pharmacists will be excluded from voting, but will participate in assessing the quality of the data for the adverse effects profile of ILE. Participants divulged all potential conflicts of interests prior to inclusion in the workgroup (Table 1) and the final selection was done to avoid conflict of interest (financial, academic or others) to respect the Institute of Medicine most recent recommendations. (13)

The first meeting of the workgroup took place in Atlanta, Georgia, USA on September 29th 2013. The majority of the members at the time were present either in person or by telephone $(\mathrm{N}=16 / 24)$; one non-voting workgroup member was added after the meeting. The purpose of this meeting was to establish the methodology structure, and agree on a timeline and work division in order to produce recommendations that will be both rigorous and transparent. Decisions on methodology were based on majority votes $(50 \%+1)$

\section{Criteria for publication inclusion}

Type of Participants

The studied participants for the evaluation of the efficacy of ILE will be patients or animals to whom ILE was given. There will be no restriction on the context of the poisoning (acute or chronic), on the poison or on the heterogeneity of the participants (e.g. special populations such as pediatric, chronic kidney disease, hepatic insufficiency, pregnancy). Studied participants for the search regarding adverse effects of ILE will include patients who received ILE either in for poisoning or for other indications. 


\section{Type of Interventions}

ILE will be considered as the intervention of interest for the efficacy evaluation only if instituted, at least partially, for the purpose of treating poisoning. Studies in which ILE was instituted for other indications will be excluded. Any administration of ILE therapy (poisonings or total parental nutrition) will be reviewed to estimate the risk of adverse events.

\section{Type of comparisons}

Clinical efficacy of ILE versus other resuscitative therapies will be reviewed. When applicable, different formulations, dosages and durations of ILE will be compared for efficacy or adverse events.

\section{Type of outcomes}

The primary outcome of interest is survival. Secondary outcomes, seizure duration, time to resolution of cardiac dysrhythmias, time and achievement of hemodynamic endpoints, (blood pressure, heart rate), neurological recovery (altered mental status, coma), and potential adverse events (pancreatitis, fat emboli, etc.) associated with ILE will also be evaluated.

\section{Type of studies}

Study designs that will be considered for inclusion in the systematic reviews are: randomized controlled trials, non-randomized controlled trials, observational studies, case series/ case reports, abstracts from clinical toxicology meetings (from 2000 onwards), abstracts from other specialties (from 2010 onwards), and animal studies for 
the different systematic reviews. For the comprehensive review addressing analytical interferences due to ILE, reports of method and instrument evaluations published in peer-reviewed journals will be appraised. Additionally, abstracts from representative clinical toxicology meetings and clinical chemistry meetings from 2009 onwards as well as package inserts from widely used and situationally-relevant assay methodologies will be included for consideration.

\section{Publication selection}

Search Strategy

Two medical librarians (MM, AMN) will identify candidate studies for this review by conducting three searches. The first will be a systematic search on ILE in toxicology for the treatment both of local anesthetic toxicity and non-local anesthetic toxicity; results from this search thus will provide the basis for two of the three systematic reviews to be produced by this workgroup. The second will be a systematic search on adverse effects of ILE and parenteral nutrition. The third search on lipid laboratory interferences will be conducted in order to provide supporting evidence during the analysis of the third systematic review, and will be thus comprehensive rather than systematic.

For each search, we will use a search strategy for Medline (via Ovid) that will be adapted to Embase (via Ovid), CINAHL (via EBSCO), BIOSIS Previews (via Ovid), Web of Science, Scopus and the Cochrane Library/DARE, with an inception date of December $15^{\text {th }} 2014$ (Appendices 2 to 4 available on-line at: insert URL here). 
The Medline search strategies will comprise a combination of Medical Subject Headings (MeSH), title/abstract key words, truncations and Boolean operators and are given in full in Appendices 2 to 4 (available on-line at: insert URL here). The first search will include the concepts of ILE and toxicology (including but not limited to calcium channel blockers, beta blockers and sodium channel blockers). The second search will include the concepts of ILE and parenteral nutrition, combined with the BMJ Clinical Evidence Adverse Effects filter (BMJ Clinical Evidence, 2012).

In addition, conference abstracts from the European Association for Poison Centres and Clinical Toxicologists, and the North American Congress of Clinical Toxicology (both from 2000-2014) and previous reviews will be hand-searched by various group members. Abstract from the Asia Pacific Association of Medical Toxicology will be searched from 2007 to 2014. Group members will perform cross-referencing of full-text articles. No limits will be applied for language and candidate studies in languages not known to any of the authors will be translated.

\section{Publication exclusion}

The workgroup will consider any original peer-reviewed article or abstract published in a peer-reviewed journal. Reviews, editorials, book chapters, and commentaries will be included only if they contained original data. Studies which after full text review will be found not to contain enough data will be excluded. Animal studies will be excluded if the methods and results are uninterpretable or cannot be extrapolated to humans. Publications describing the use of ILE solely for the treatment of nutritional needs will 
be excluded from the efficacy systematic review, but will be included in the adverse effect systematic review. Pre-treatment studies and experimental in-vitro or ex-vivo models will be excluded due to their inability to be generalizable to the context of human poisoning.

Reporting by subgroup

The workgroup will be divided into subgroups, each of which will be responsible for reviewing a different aspect of the therapy. A summary of the search strategy will be described in their distinct manuscript for each systematic review by the responsible subgroup and the librarians, according to the PRISMA statement (14). It will describe in detail the number of references retrieved in the initial search. The number of duplicates and excluded publications will be stated, with the reason for exclusion (relevance, very poor quality, publication type). Any disagreement in the exclusion process will be addressed explicitly.

\section{Poison selection}

All poisons for which ILE will be reported as a treatment for acute or chronic toxicity will be included for the systematic review. Poisons for which the group will determine that sufficient cases have been reported (more than 3 subjects) will be evaluated through the voting process, which is to occur at a later date.

\section{Data extraction, synthesis, presentation and interpretation}

Data extraction 
For each publication retained in each different database, the responsible subgroup will extract all relevant data into a standardized data extraction document. Relevant data that will be extracted will include : demographic information, description of poisoning (amount of poison, timing, administration route, coingestion), severity of poisoning (poison specific clinical symptoms, signs and relevant laboratory analyses), ILE administered (lipid formulation and concentration, use of a bolus (amount and timing of administration), use of an infusion (rate and duration) and total amount of lipid received converted in $\mathrm{g} / \mathrm{kg}$ ), concomitant treatments (type, dose and duration), clinical outcomes (general and specific to the described poisoning), adverse effects of therapy (type, severity and timing) and duration of follow-up. At this stage, no calculation, inference or interpretation will be attempted. All publications likely to be excluded (according to the previous criteria) will be marked for re-evaluation by the expert subgroup. To reduce the risk of errors, two independent reviewers will evaluate each publication. To ensure uniformity, the group leader will merge individual data extraction flow sheets. If observational studies or randomized controlled trials are included, the epidemiologist will assist the evaluation of the quantitative measure of effect and quality of evidence for clinical outcomes.

\section{Evaluation of extracted data}

For each publication included in the specific poison database, the subgroup will first evaluate publications marked for exclusion. A publication will be rejected only if all members of the subgroup agree. If there is strong disagreement on the inclusion or exclusion of a determined publication, the chair will adjudicate. When the final summaries will be presented to the workgroup for the recommendations assessment, 
the arguments for and against inclusion of a particular publication will also be presented for transparency. Each individual publication will be assessed in regards to its quality for clinical outcomes or adverse effects accordingly with recognized reporting guidelines (15-20). The final level of evidence will be reported as per the GRADE system

(methodological biases, indirectness, imprecision, and error) $(11,21)$. Also, the effect of ILE will be reported for each clinical outcome (descriptive value and/or comparative values, such as risk difference, relative risk or p-value, if applicable). Because of the controversy as to which kinetic outcome (free drug concentration or apparent half-life changes) is valid to appraise success of ILE, they will only be reported if measured.

\section{Data Synthesis}

Following the previous steps, the subgroup will create a summary sheet. Data will be regrouped to allow synthesis principally by poison or group of poisons, and if feasible, by intervention and by sub-population. The quality of the evidence will be summarized for each outcome (Appendix 5 available on-line at: insert URL here). The effect of ILE and risk of adverse effects will be summarized qualitatively or quantitatively for each outcome. For the review of analytical interferences, the data will be grouped by analyte and/or methodology. Any general comments on the reviewed literature will be added to the summary sheet. The definitions and terminology used is presented in Table 2 .

\section{Lipid emulsion costs}

A survey will be distributed internationally to pharmacists of health care agencies and health care organizations to assess the acquisition costs and patient charges for different ILE formulations and comparative treatments available in their countries. This is not 
intended to provide a rigorous cost analysis study or to assess cost-effectiveness of ILE. Rather, it will give an overall idea of the monetary costs associated with administration of various ILE formulations, their adequate storage and expiry date required for availability.

\section{Recommendations on the use of ILE}

\section{Statement proposal}

After reviewing and summarizing the available literature, the subgroup will propose a series of statements concerning the use of ILE in poisoning. These statements will take into account the quality of evidence, the relative importance of the outcomes, expected clinical course without ILE, the availability of other therapies, the magnitude and the precision of the effect, the balance between benefits and harms and the costs of the procedure.

\section{Prototype Statements}

1) General statement: (we recommend/ we suggest/ neutral position/ no agreement reached) to (give/ not give) ILE in poisoning with "X".

2) Specific statements: If there is support, other statements will be submitted:

indications for initiating ILE (ingestion dose, drug concentration, special population, symptoms, clinical markers), type of ILE regimen (dose, duration, rate and formulation of ILE), and any other statement particular to the poison deemed significant by the subgroup (e.g. alternative therapy, antidote). 
The subgroup will submit the following documents to the workgroup: complete publications, merged flow sheet, summary sheet and the proposed voting statements. At any step throughout the process, if there is strong disagreement or dissent, the issues will be brought to the entire workgroup for review.

\section{Voting procedure}

Since much of the literature reviewed is likely to be of low methodological quality, the majority of recommendations will be based on a consensus of expert opinions. Therefore, a rigorous voting procedure will be implemented to ensure transparency and reproducibility. The modified Delphi method (i.e., an iterative consultation of experts on a given subject) was chosen to reach a formal consensus on proposed voting statements. Two rounds of consultation are scheduled before the final version is submitted.

For the first round, the subgroup will submit statements to each workgroup member who anonymously will indicate their level of agreement and include comments. Approval for the proposed statement will be recorded on a 9-point Likert scale (with 1 being completely against and 9 being completely for the proposed statement). Every member will be encouraged to expand on or challenge proposed statements.

After completion of the first round, votes and comments will be summarized. The RAND/UCLA Appropriateness Method (a method of statistical measurement frequently used and adapted to all panel sizes) will be used to quantify the votes (22). The median values and the lower/upper quartiles will be reported and the disagreement indexes calculated. Median values ranging from 7-9 will reflect that the workgroup is in favor of 
the proposed statement, 4-6 will reflect a neutral position and 1-3 will reflect that the workgroup is not in favor of the statement. The disagreement index, defined as the interpercentile range divided by the interpercentile range adjusted for symmetry, describes the dispersion of ratings more effectively than the mean absolute deviation from the median. Index values less than or equal to 1 will indicate agreement (23).

A standardized form will then be resubmitted to each participant with his or her vote, summary statistics, and workgroup comments and modified statements. The second voting round will take place after a meeting where the results of the first round of the votes will be discussed in a face-to-face meeting for the majority of the workgroup members and others joining by teleconferencing. All subgroups will present the evidence a second time i.e. potential risks, costs, alternative treatments, clinical benefit, for every reviewed poison. After this presentation, the workgroup will take time to deliberate these findings. Each statement will then be re-voted privately and anonymously.

The voting procedure will result in providing strength of recommendations (see Figure 1). Because of the restricted number of experts voting, if dissent remains, the final decision and debate will be explained in the official recommendation document for transparency. Interpretation of each level of strength of recommendation is explained on Table 3.

\section{Values and preferences}


Values and preferences of the patients will not be evaluated as part of this work (after discussion with a representative of the AGREE consortium) since this is not a homogenous population. The clinical situations evaluated being in a resuscitative context pose inherent limitations to the timeliness of decision-making by clinicians and the ability of patients to evaluate their own risk-benefit considerations. However, values and preferences susceptible to influence the votes of the stakeholders will be evaluated at the end of the process by a survey conducted by the methodologist.

\section{Writing and review process}

The Board of Trustee of the American Academic of Clinical Toxicology (AACT), as the main sponsoring organization, has first endorsed this methodology manuscript, while other participating organizations provided comments through their representatives. Four distinct manuscripts will be prepared for the evidence reviews and will be submitted through standard peer-reviewed publication process. No supplementary review or endorsement will be required by AACT for these scientific reviews. The last manuscript which is to include the recommendations will be submitted to AACT and other participating organizations for endorsement prior to publication.

\section{Implementation and applicability}

Following endorsement by AACT and publication of the recommendations, these recommendations will be available on AACT website for dissemination. Members of the workgroup intend to conduct an international survey of end-user clinicians to identify the impact of the recommendations on their practice. 


\section{Updating}

These recommendations will be updated if there is a significant change in the evidence, at the request of AACT or no later than 5 years after publication. A search strategy will be registered in different search engines for updates.

\section{Conclusion}

Intravenous lipid emulsion therapy for poisoning is a recent development in medical toxicology. The heterogeneity of the literature warrants a thorough review and appraisal of the evidence. Rigorous methodology combined with a transparent recommendations process development will assist clinicians in making choices when treating patients with substances potentially amenable to treatment with intravenous lipid emulsion therapy.

\section{Acknowledgments}

The workgroup wishes to thank Ms Ellen Pak and Ms Sarah Shiffert for their administrative assistance in organizing meetings.

\section{Conflict of interest}

The authors report no conflict of interest related to this work.

\section{Funding}

Participants did not receive honoraria. The American Academic of Clinical Toxicology provided funding for conference calls. Dr Turgeon and Dr Lavergne are recipients of a career award from the Fonds de Recherche du Québec - Santé (FRQS). 


\section{References}

1. Sites BD, Taenzer AH, Herrick MD, Gilloon C, Antonakakis J, Richins J, Beach ML. Incidence of local anesthetic systemic toxicity and postoperative neurologic symptoms associated with 12,668 ultrasoundguided nerve blocks: an analysis from a prospective clinical registry. Regional anesthesia and pain medicine. 2012;37(5):478-82.

2. Mowry JB, Spyker DA, Cantilena LR, Jr., Bailey JE, Ford M. 2012 Annual Report of the American Association of Poison Control Centers' National Poison Data System (NPDS): 30th Annual Report. Clinical toxicology. 2013;51(10):949-1229.

3. Weinberg GL, VadeBoncouer T, Ramaraju GA, Garcia-Amaro MF, Cwik MJ. Pretreatment or resuscitation with a lipid infusion shifts the dose-response to bupivacaine-induced asystole in rats. Anesthesiology. 1998;88(4):1071-5.

4. Rosenblatt MA, Abel M, Fischer GW, Itzkovich CJ, Eisenkraft JB. Successful use of a $20 \%$ lipid emulsion to resuscitate a patient after a presumed bupivacaine-related cardiac arrest. Anesthesiology. 2006;105(1):217-8.

5. Sirianni AJ, Osterhoudt KC, Calello DP, Muller AA, Waterhouse MR, Goodkin MB, Weinberg GL, Henretig FM. Use of lipid emulsion in the resuscitation of a patient with prolonged cardiovascular collapse after overdose of bupropion and lamotrigine. Ann Emerg Med. 2008;51(4):412-5, 5 e1.

6. American College of Medical T. ACMT Position Statement: Interim Guidance for the Use of Lipid Resuscitation Therapy. Journal of Medical Toxicology. 2011;7(1):81-2.

7. Jamaty C, Bailey B, Larocque A, Notebaert E, Sanogo K, Chauny JM. Lipid emulsions in the treatment of acute poisoning: A systematic review of human and animal studies. Clinical toxicology. 2010;48(1):1-27.

8. Cave G, Harvey M, Graudins A. Review article: Intravenous lipid emulsion as antidote: A summary of published human experience. EMA - Emergency Medicine Australasia. 2011;23(2):123-41.

9. Waring WS. Intravenous lipid therapy: A systematic review of published case reports. Clinical toxicology. 2012;50 (4):365-6.

10. Butterworth JFt. Models and mechanisms of local anesthetic cardiac toxicity: a review. Regional anesthesia and pain medicine. 2010;35(2):167-76.

11. Guyatt GH, Oxman AD, Kunz R, Vist GE, Falck-Ytter Y, Schunemann HJ. What is "quality of evidence" and why is it important to clinicians? BMJ. 2008;336(7651):995-8.

12. Brouwers MC, Kho ME, Browman GP, Burgers JS, Cluzeau F, Feder G, Fervers B, Graham ID, Grimshaw J, Hanna SE, Littlejohns P, Makarski J, Zitzelsberger L, Consortium ftANS. AGREE II: advancing guideline development, reporting and evaluation in health care. Canadian Medical Association Journal. 2010;182(18):E839-E42.

13. Institute Of Medicine. Clinical Practice Guidelines We Can Trust. Washington DC: National Academies Press, 2011.

14. Moher D, Liberati A, Tetzlaff J, Altman DG, Group P. Preferred reporting items for systematic reviews and meta-analyses: the PRISMA statement. BMJ. 2009;339:b2535.

15. Schulz KF, Altman DG, Moher D, Group C. CONSORT 2010 statement: updated guidelines for reporting parallel group randomised trials. BMJ. 2010;340:c332.

16. von Elm E, Altman DG, Egger M, Pocock SJ, Gotzsche PC, Vandenbroucke JP, Initiative S. The Strengthening the Reporting of Observational Studies in Epidemiology (STROBE) statement: guidelines for reporting observational studies. J Clin Epidemiol. 2008;61(4):344-9.

17. Gagnier JJ, Riley D, Altman DG, Moher D, Sox H, Kienle G, Group C. The CARE guidelines: consensusbased clinical case reporting guideline development. Dtsch Arztebl Int. 2013;110(37):603-8.

18. Lavergne V, Ouellet G, Bouchard J, Galvao T, Kielstein JT, Roberts DM, Kanji S, Mowry JB, Calello DP, Hoffman RS, Gosselin S, Nolin TD, Goldfarb DS, Burdmann EA, Dargan PI, Decker BS, Hoegberg LC, Maclaren R, Megarbane B, Sowinski KM, Yates C, Mactier R, Wiegand T, Ghannoum M. Guidelines for reporting case studies on extracorporeal treatments in poisonings: methodology. Semin Dial. 2014;27(4):407-14. 
19. Kilkenny C, Browne WJ, Cuthill IC, Emerson M, Altman DG. Improving bioscience research reporting: the ARRIVE guidelines for reporting animal research. PLoS Biol. 2010;8(6):e1000412.

20. Kelly WN, Arellano FM, Barnes J, Bergman U, Edwards RI, Fernandez AM, Freedman SB, Goldsmith DI, Huang KA, Jones JK, McLeay R, Moore N, Stather RH, Trenque T, Troutman WG, van Puijenbroek E, Williams F, Wise RP, International Society for P, International Society of P. Guidelines for submitting adverse event reports for publication. Drug Saf. 2007;30(5):367-73.

21. Guyatt $G H$, Oxman $A D$, Kunz $R$, Jaeschke $R$, Helfand $M$, Liberati $A$, Vist GE, Schunemann HJ. Incorporating considerations of resources use into grading recommendations. BMJ. 2008;336(7654):1170-3.

22. Fitch K, Bernstein SJ, Aguilar MD, Burnand B, LaCalle JR, Lazaro P, Loo MVH, McDonnell J, Vader JP, Kahan JP. The RAND/UCLA Appropriateness Method User's Manual. Santa Monica, CA2011. Available from: http://www.rand.org/.

23. Shiffman RN, Shekelle P, Overhage JM, Slutsky J, Grimshaw J, Deshpande AM. Standardized reporting of clinical practice guidelines: a proposal from the Conference on Guideline Standardization. Ann Intern Med. 2003;139(6):493-8. 